\title{
Effect of Nozzle Lip Shape on Screech Tone in a Supersonic Jet
}

\author{
By Yongseok KIM ${ }^{1)}$ and Yoshiaki NAKAMURA ${ }^{2)}$ \\ ${ }^{1)}$ School of Mechanical and Aerospace Engineering, Sunchon National University, Suncheon, Korea \\ ${ }^{2)}$ Department of Aerospace Engineering, Nagoya University, Nagoya, Japan
}

(Received October 23rd, 2006)

\begin{abstract}
The effect of nozzle lip shape on screech tone is investigated by a computational aeroacoustic approach. First, the scattered patterns of screech tone are examined without a jet mean flow, where plane waves interact with a nozzle lip wall. Then, an axisymmetric jet screech is simulated in the Mach number range from 1.07 to 1.2, where the axisymmetric mode is a dominant screech mode. The conservative form of the axisymmetric Euler equations written in generalized coordinates are used to treat the complex nozzle lip geometry for the scattering problem as well as the Reynolds Averaged Navier-Stokes equations with the modified Spalart-Allmaras turbulence model. The computed shock-cell structure, screech tone frequency, and sound pressure levels in the near field are in good agreement with existing experimental data.
\end{abstract}

Key Words: Supersonic Jet, Screech Tone, Nozzle Lip Shape, ENO Scheme, Noise Scattering

\section{Introduction}

Most high-speed jets accompanying propulsion systems produce intense radiated jet noises. In rare cases, the acoustic waves can cause a structural failure of the propulsion system by resonant interference with the structure as well as failure of the payload inside the structure. Since, in civil aircraft, noise levels near airports are restricted, reduction in near-ground operation noises is an important issue for aircraft and engine manufactures. Therefore, noise generation has now become an important design factor in the early construction process. Imperfectly expanded supersonic jets emit mixing noise and broadband shock-associated noise as well as screech tone under certain conditions.

The screech tone is known to be generated by a feedback loop. Better understanding of the screech feedback mechanism and better prediction of the screech amplitude are needed to suppress screech tones without loss of performance. The main part of screech tones generated by shockinstability interactions propagate upstream and impinge on the nozzle lip wall, and the reflected and scattered waves interact with the thin shear layer of the jet. This process amplifies the instability waves, leading to generation of strong screech tones. To identify the feedback-loop mechanism more clearly and predict the amplitude of screech tones more accurately, both experimental and numerical studies have been made, including the pioneering work by Powell ${ }^{1)}$ and many other researchers. ${ }^{2-5)}$

The objective of the present paper is to examine how the nozzle lip shape affects the screech tone. To analyze the scattered pattern by screech tones, the acoustic scattering problem regarding nozzle lip is taken first, where jet mean flow is not considered. This scattering problem can be realized by the interaction between plane waves, which represent screech tones, and a nozzle lip wall. From this

C) 2008 The Japan Society for Aeronautical and Space Sciences simulation, an optimal nozzle lip shape can be found to minimize the reflected and scattered sound waves, which will lead to reduction in screech tones in the supersonic jet. The latter thing is examined by simulating actual screech jet flow. To do this, a high-resolution, finite volume essentially non-oscillatory (ENO) scheme is used along with nonreflecting boundary conditions that are crucial to accurately capture the amplitude and directivity of generated sounds with regard to screech tone propagation and scattering.

The next section describes the governing equations, turbulence model, numerical methods, and boundary conditions. Detailed numerical validations and results are presented in section 3 , and the conclusion is given in section 4 .

\section{Governing Equations and Numerical Methods}

\subsection{Governing equations}

The axisymmetric mode is a dominant screech mode for axisymmetric supersonic jets at low Mach numbers. In this study, to treat this mode, the axisymmetric RANS equations are used with the modified Spalart-Allmaras turbulence model $^{6)}$ in the Mach number range from 1.07 to 1.2 . On the other hand, the pure scattering problem is realized by the interaction between plane waves, which represent screech tones, and a nozzle lip wall. The conservative form of the axisymmetric Euler equations written in generalized coordinates is used for this problem.

The Spalart-Allmaras model belongs to the family of eddy viscosity models. This kind of model is based on the assumption that the Reynolds stress tensor $\left(-\rho \overline{u_{i} u_{j}}\right)$ is linearly related to the mean flow strain rate tensor through the apparent turbulent viscosity called eddy viscosity $v_{\mathrm{T}}$. In the Spalart-Allmaras model, the eddy viscosity is obtained by solving the partial differential equation for intermediate variable $\bar{v}$, in which $v_{\mathrm{T}}$ is related to $\bar{v}$ through as followings: 


$$
v_{\mathrm{T}}=\bar{v} f_{\mathrm{v} 1}(\chi), \quad \chi=\frac{\bar{v}}{v}
$$

where $f_{\mathrm{v} 1}$ is a damping function.

The differential transport equation for $\bar{v}$ is written as follows:

$$
\begin{aligned}
\frac{D \bar{v}}{D t}= & \frac{1}{\sigma}\left[\nabla \cdot\left((\nu+\bar{v}) \nabla \bar{v}+c_{\mathrm{b} 2}(\nabla v)^{2}\right]+c_{\mathrm{b} 1} \bar{S} \bar{\nu}\left(1-f_{\mathrm{t} 2}\right)\right. \\
& -\left[c_{\mathrm{w} 1} f_{\mathrm{w}}-\frac{c_{\mathrm{b} 1}}{\kappa^{2}} f_{\mathrm{t} 2}\right]\left[\frac{\bar{v}}{d}\right]^{2}+f_{\mathrm{t} 1}(\Delta q)^{2}
\end{aligned}
$$

The various functions and constants are reported in the references. ${ }^{7)}$ In the present study, the following values are used.

$$
\begin{aligned}
& \sigma=2 / 3, \quad c_{\mathrm{b} 1}=0.1355, \quad c_{\mathrm{b} 2}=0.622 \\
& c_{\mathrm{w} 1}=\frac{c_{\mathrm{b} 1}}{\kappa^{2}}+\left(1+c_{\mathrm{b} 2}\right) / \sigma, \quad c_{\mathrm{w} 2}=0.3, \quad c_{\mathrm{w} 3}=2, \quad \kappa=0.41 \\
& c_{\mathrm{v} 1}=7.1, \quad c_{\mathrm{t} 1}=1.0, \quad c_{\mathrm{t} 2}=2.0, \quad c_{\mathrm{t} 3}=1.1, \quad c_{\mathrm{t} 4}=2.0
\end{aligned}
$$

Note that the trip function, $f_{\mathrm{t} 1}$, and the function $f_{\mathrm{w}}$ are neglected in the present simulation. In addition, the coefficient, $c_{\mathrm{b} 1}$, is adjusted depending on screech jet Mach number.

\subsection{Numerical algorithm}

Both a high-resolution numerical scheme and appropriate boundary treatment are crucial for accurate computation of aeroacoustic problems, especially for screech tone simulation. The screeching jet contains sound waves, large-scale instability waves and shock cells, which produce a wide range of pressure amplitude. This study uses the finite volume MFA(Modified-Flux-Approach)-ENO (Essentially Non-oscillatory) scheme along with the characteristic boundary conditions to obtain periodic solutions in time, based on screech cycles. The MFA-ENO scheme considered here has been verified by a geometrically complicated system, ${ }^{8)}$ where the uniform 4th-order accuracy in time and space has been achieved both at local extrema of the solutions and in other smooth regions. Not only strong disturbances, such as a shock-cell and a large-scale vortex, but also the small amplitude of sound waves, can be accurately resolved. ${ }^{9)}$

\subsection{Initial and boundary conditions}

As shown in Fig. 1, Thompson's characteristic boundary conditions ${ }^{10)}$ are used as non-reflective boundary conditions at the left boundary and in the outer boundary regions. Based on Thompson's approach, physical boundary conditions for computation are derived by using characteristic variables. Detailed derivation of the non-reflective boundary conditions can be found in the references. ${ }^{11)}$ In the downstream boundary region where $M_{\mathrm{a}} \geq 0.001$, the outflow boundary conditions are implemented. A buffer zone is used to reduce the vortex strength passing through the outflow boundary eliminating the numerical reflections.

At the nozzle exit, the flow variables are taken to be uniform corresponding to those at the exit of a convergent nozzle. The jet operating conditions are fully determined by the reservoir to ambient pressure and temperature ratios.

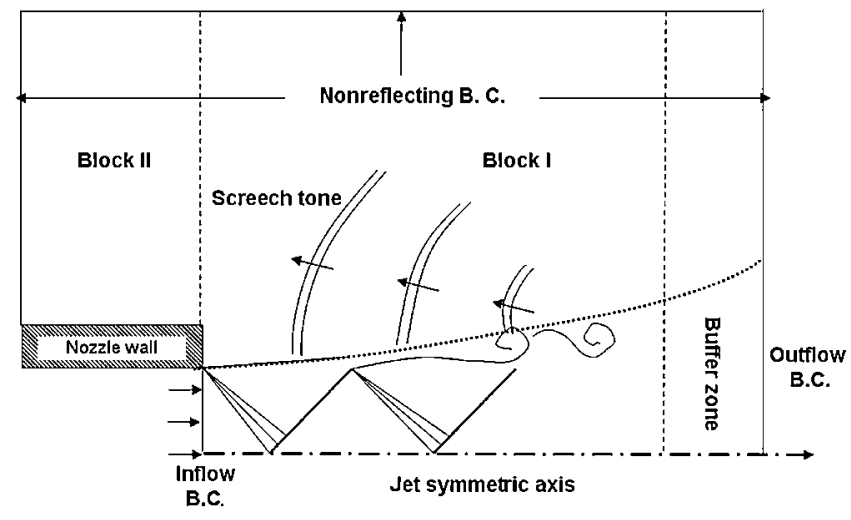

Fig. 1. Schematic diagram and boundary conditions for screech jet simulation.

The pressure ratio is commonly expressed in terms of the fully expanded jet Mach Number $M_{\mathrm{j}}$. All nondimensional variables are given as follows:

$$
\begin{aligned}
\rho_{\mathrm{e}} & =\frac{\gamma(\gamma+1) p_{\mathrm{e}}}{2 T_{\mathrm{r}}} \\
P_{\mathrm{e}} & =\frac{2}{\gamma}\left[\frac{2+(\gamma-1) M_{\mathrm{j}}^{2}}{\gamma+1}\right]^{\frac{\gamma}{\gamma-1}} \\
u_{\mathrm{e}} & =\left(\frac{2 T_{\mathrm{r}}}{\gamma+1}\right)^{1 / 2}, \quad v_{\mathrm{e}}=0
\end{aligned}
$$

where $\gamma$ is equal to 1.4. $T_{\mathrm{r}}$ is the reservoir temperature, which is set to 1 for the cold jet assumption.

In the turbulence model, the turbulent viscosity at the nozzle exit is set to zero.

\section{Numerical Results}

\subsection{Screech tone in supersonic jet}

\subsubsection{Mean velocity profile and shock-cell structure}

An axisymmetric supersonic jet is simulated at a fully expanded jet Mach number range between 1.07 and 1.2 to ensure generation and propagation of axisymmetric screech tone modes. For verification, the simulated mean flows were compared with experiments. Specifically, the time-averaged axial velocity profiles were computed for a Mach 1.2 jet from one diameter downstream of the nozzle exit to six diameters, which is shown in Fig. 2. The results are plotted as a function of $\eta^{*}=\left(r-r_{0.5}\right) / x$, where $r_{0.5}$ is the radial distance from the jet axis to the location where the axial velocity is equal to half of the fully expanded jet velocity. Numerous experimental measurements have shown that the mean velocity profiles almost collapse into a single curve with regard to the similarity variable $\eta^{*}$. Extensive data were measured by Lau, ${ }^{12)}$ and Lau's empirical formula is also plotted in Fig. 2. There is good agreement.

Figure 3 shows the time-averaged pressure along the jet axis at $M_{\mathrm{j}}=1.2$. One important issue for screech tone is shock-cell structure. Therefore, the shock-cell spacing and shock amplitude must be calculated accurately to ensure the accuracy of both screech frequency and amplitude. The numerical result is compared with the experimental data 


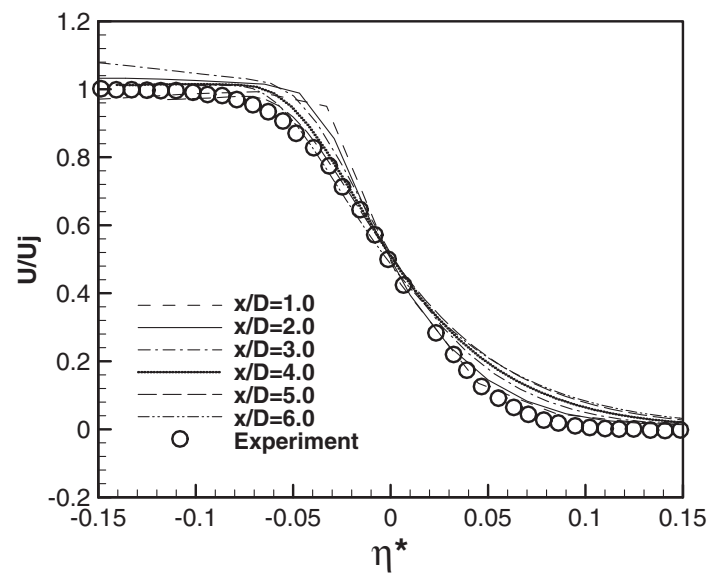

Fig. 2. Comparison between mean velocity profiles of numerical simulation and experimental data ${ }^{12)}$ at $M_{\mathrm{j}}=1.2$.

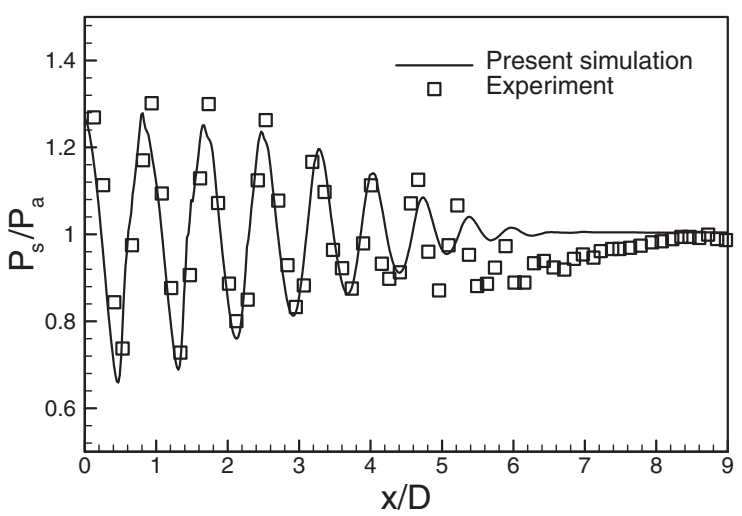

Fig. 3. Centerline pressure comparison between present calculation and experimental data by Norum \& Brown ${ }^{13)}$ for cold jet at $M_{\mathrm{j}}=1.2$.

by Norum \& Brown. ${ }^{13)}$ The first five shocks show good agreement.

The first three shock cells are almost steady, while the following shock cells are unsteady due to convection of largescale vortices. We examined this phenomenon in detail through the Mach number variation in the jet plume in Fig. 4. The shock cells after the fourth one move back and forth, and the flow structure changes definitely during the screech cycle T. In order to see the more detailed unsteady motion of the shock cells, Mach number variation is plotted with six equal intervals during a cycle. The mechanism of screech generation can be explained by the periodic motion of the shock-cell structure. Lines I to VII represent the shock-cell locations, and the fourth (IV) and fifth (V) shock cells are compressed from $t=t_{1}$ to $t=t_{1}+3 T / 6$ and expanded from $t=t_{1}+4 T / 6$ to $t=t_{1}+T$, as seen in Fig. 4(a). This periodic compression and expansion of an air flow generates acoustic waves such as screech tone.

Figure 5 shows density contours representing the shockcell structures and unsteady shock motions in the supersonic jet core region. The first three shock cells are almost steady, but after the fourth shock cell, unsteady shock motions interact with instability waves, causing compression and expansion of the jet flow and producing screech tone.
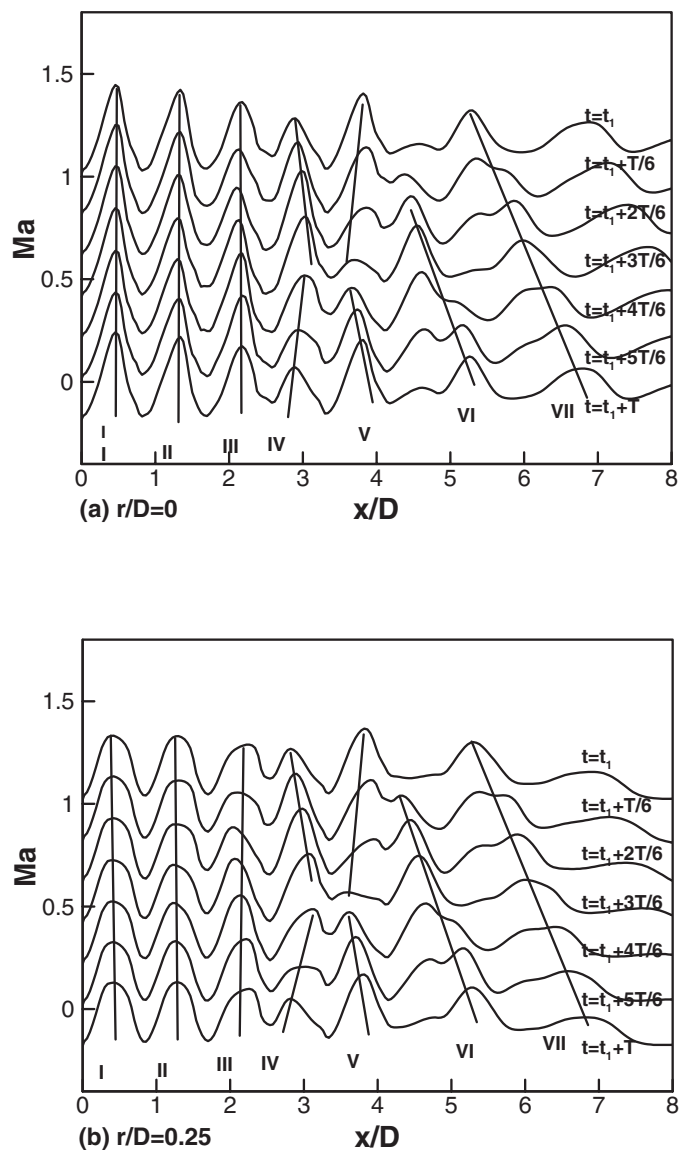

(b) $r / D=0.25$

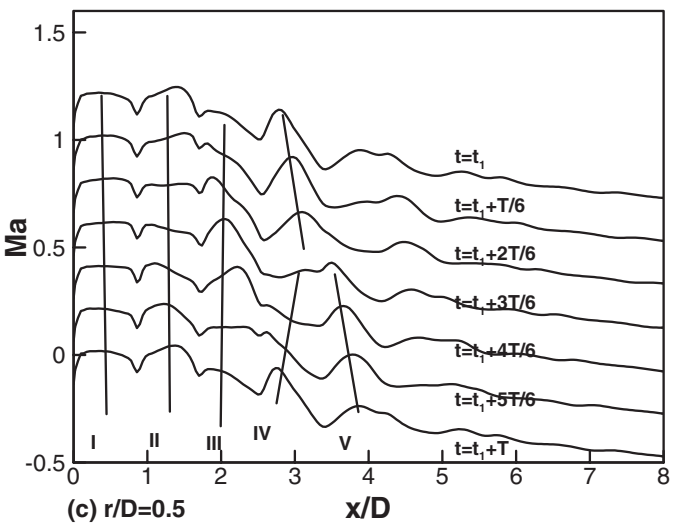

Fig. 4. Mach number variations at several radial locations during screech cycle.

\subsubsection{Screech tone frequency}

It is well known that there are two axisymmetric modes: $A_{1}$ and $A_{2}$ modes at low supersonic jet Mach number. However, the mode transition phenomenon has not been clarified. In the present numerical simulation, both the $A_{1}$ and $A_{2}$ axisymmetric modes are well captured. Numerical results for the screech tone wavelength at different Mach numbers ranging from 1.07 to 1.2 are shown in Fig. 6 along with the experimental data of Ponton and Seiner. ${ }^{14)}$ They are in good agreement, and collapse on two curves: one for the $A_{1}$ mode and the other for the $A_{2}$ mode. However, the critical Mach number where the mode jumps from $A_{1}$ to 

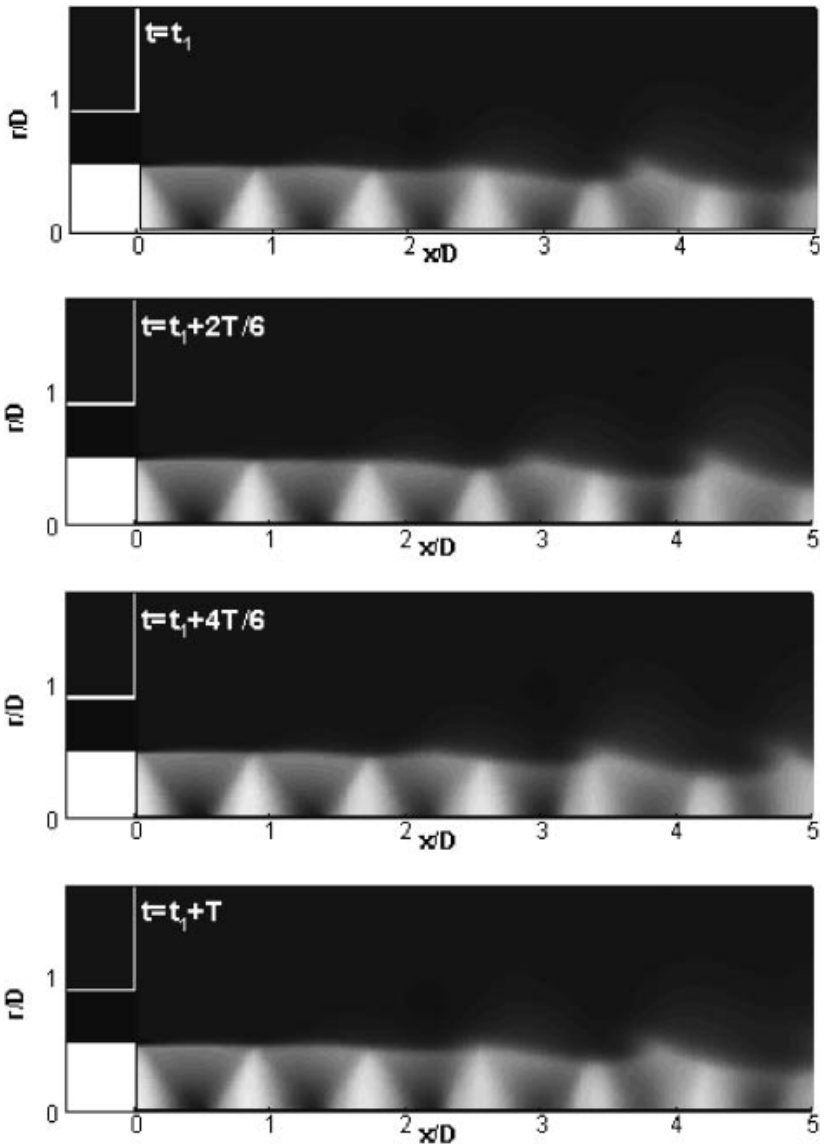

Fig. 5. Density contours showing shock cell structures and unsteady shock motions in supersonic jet core region.

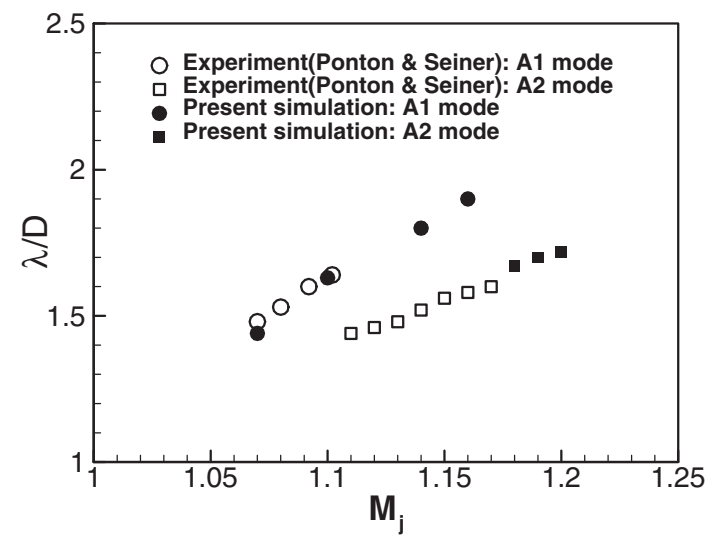

Fig. 6. Comparison of screech tone wavelength between present simulation and experimental data of Ponton and Seiner. ${ }^{14)}$

$A_{2}$ is not the same. According to Norum, ${ }^{15)}$ this critical Mach number as well as its frequency is very sensitive and varies from experiment to experiment.

\subsection{Screech scattering problem}

This section deals with a screech scattering problem to examine the effect of nozzle lip shape on the reflected and scattered fields. The screech tones generated by shock-instability interactions mainly propagate upstream and impinge on the nozzle lip wall, where the reflected and scattered waves interact with the thin shear layer of the jet. This proc-

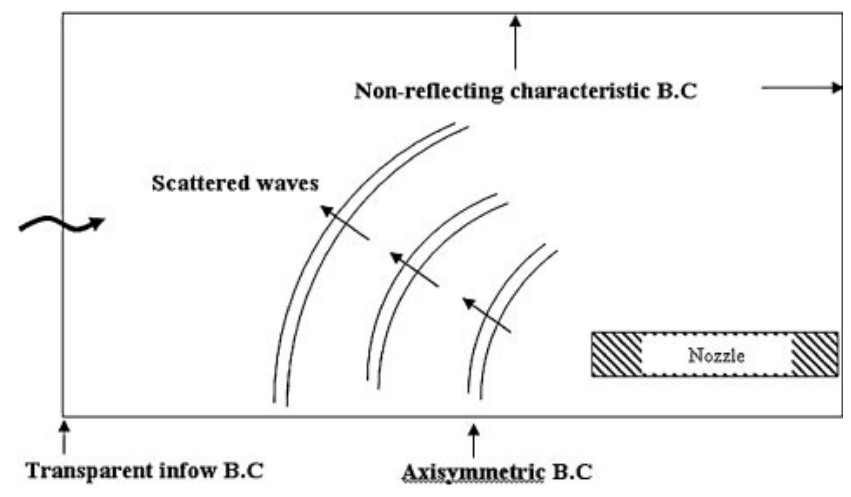

Fig. 7. Schematic diagram of screech tone scattering simulation.

ess amplifies the instability waves, leading to strong screech tones.

This scattering problem can be simplified as the interaction between plane waves representing screech tones, and a nozzle lip wall. As the present study focuses on axisymmetric modes of the screech tone, the conservative form of the axisymmetric Euler equations written in generalized coordinates is used. The computational domain and boundary conditions are shown schematically in Fig. 7. The plane waves are provided from the bottom left inlet under the nonreflecting transparent characteristic boundary condition that enables the source to be transparent and prevents the plane waves from being reflected at the inlet boundary. The symmetry boundary condition is used at the jet axis.

In the present simulation, the screech wavelength and sound pressure level are set to be $1.6 D$ ( $D$ is nozzle diameter), and $135 \mathrm{~dB}$, respectively. The incident plane waves with sound pressure, $p_{i}$, have frequency, $f$, and pressure amplitude, $p_{0 \mathrm{i}}$, calculated as:

$$
p_{\mathrm{i}}=p_{0 \mathrm{i}} \Re\left[e^{i(2 \pi f t)}\right]
$$

where $\Re[z]$ denotes the real part of complex number $z$. These sound pressure waves are emitted from the bottom left corner of the computational domain, as mentioned earlier, which interacting with the nozzle lip wall after propagating some distance. The scattering process is usually characterized by incident wave, $p_{\mathrm{i}}$, total pressure, $p_{\mathrm{t}}$, and scattered wave pressure, $p_{\text {scat }}$. $p_{\text {scat }}$, can be computed from $p_{\mathrm{t}}$ and $p_{\mathrm{i}}$ as:

$$
p_{\text {scat }}=p_{\mathrm{t}}-p_{\mathrm{i}}
$$

The incident, total and scattered fields are accurately resolved by the present numerical method (Fig. 8). The nonreflecting transparent boundary condition at the inlet where reflection of the acoustic waves is not observed also works well.

We employed two different shapes as nozzle lip geometry to compare their scattered patterns. The first is a base shape, called as shape-A; the second case is an upward-cut shape, called as shape-B. The reflected and scattered patterns are clearly shown in the scattered pressure field in Fig. 8(c). In both cases, the scattered directivity is upstream dominant, 

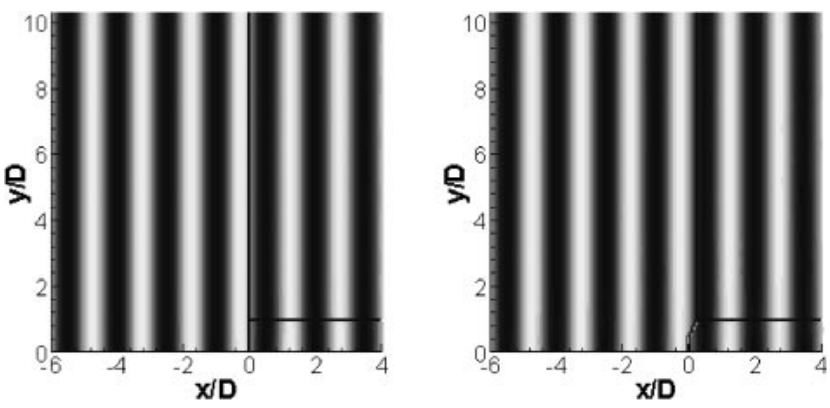

(a) Instantaneous incident pressure fields
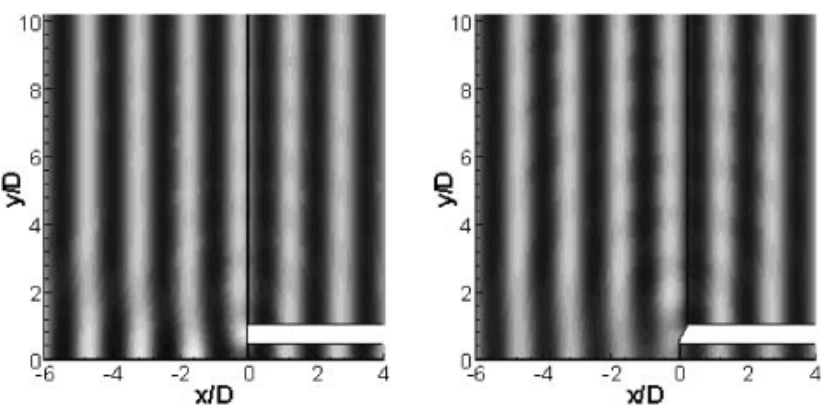

(b) Instantaneous total pressure fields
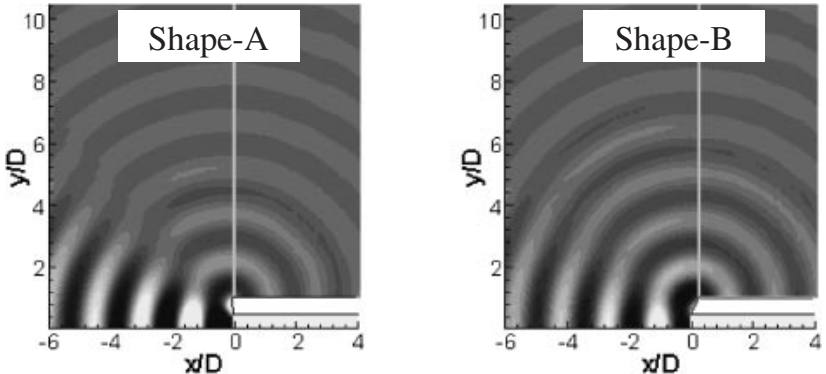

(c) Instantaneous scattered pressure fields

Fig. 8. Instantaneous pressure field in screech tone scattering problem (Shape-A: base geometry, Shape-B: upward-cut geometry).

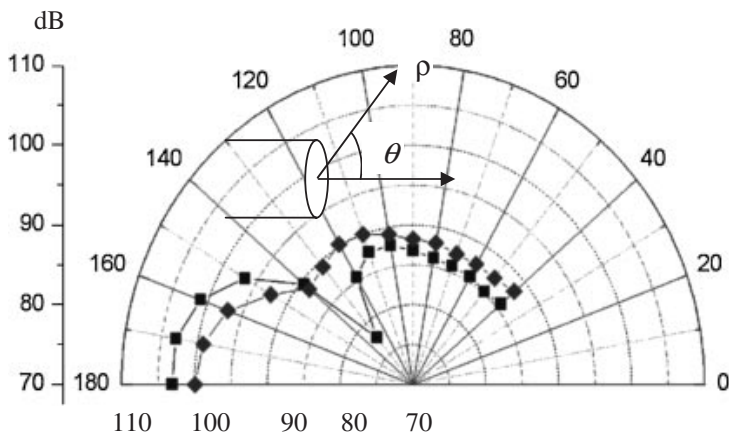

Fig. 9. Sound pressure level at $r=5 D$ with $\theta$ as parameter (Nozzle lip shape-A: $\boldsymbol{\square}$, shape-B: $\bullet$ )

but the intensity for shape-B is lower than that for shape-A, suggesting that the amplitude of reflected and scattered waves along the jet axis can be reduced by choosing an appropriate nozzle shape. To observe the difference between shape-A and shape-B in more detail, the sound pressure levels at $r=5 D$ are compared in Fig. 9. For shape-B, the

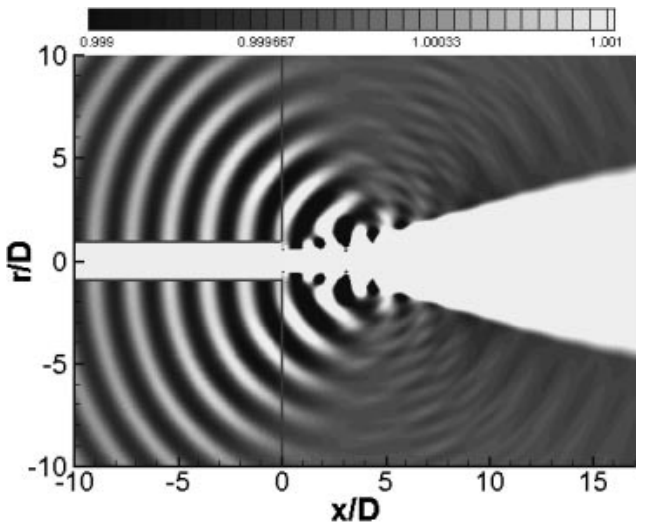

(a) Shape-A: Base geometry

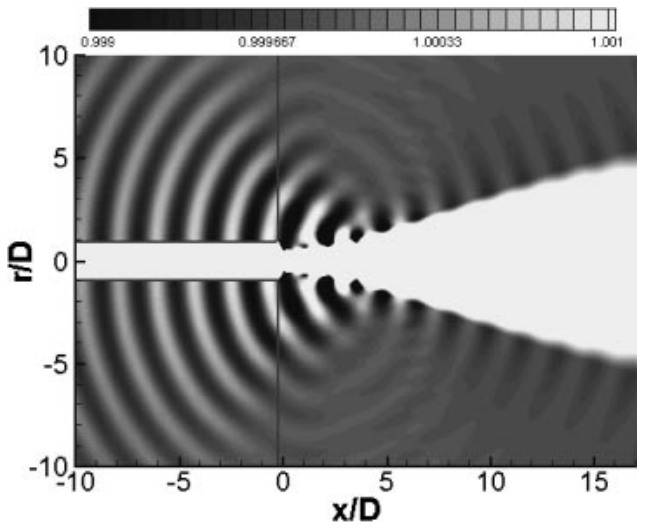

(b) Shape-B: Upward-cut geometry

Fig. 10. Comparison of instantaneous jet flow density contours between two nozzle lip shapes.

reflected part of the scattered field is suppressed along the jet axis, and the scattering waves propagate more intensively in the upper direction. This result suggests that the jet shear layer near the lip is less affected, leading to suppression of screech in real supersonic jet flows.

\subsection{Effect of nozzle lip shape on screech tone}

Here we describe calculated results for actual screeching jet flows, where two nozzle lip shapes mentioned above are used to examine the effect of nozzle lip shape on screech tone. Figures 10 and 11 compare the instantaneous density field between shape-A and shape-B at $M_{\mathrm{j}}=1.2$. The computed density contours at one instance are obtained after the initial transient patterns have propagated out of the computational domain. As seen in Fig. 10, the screech tones radiate principally in the upstream direction and interact with the nozzle lips. The acoustic disturbances impinging on the nozzle lip, where the jet mixing layer is thin and receptive to external disturbances, excite the jet shear layer, and induce additional acoustic waves. Sound waves of the screech tone are generated in the fourth to fifth shock-cell region, which propagate in the upstream direction. The difference in the magnitude and directivity of these waves between shape-A and shape-B is seen from the density contours in Fig. 10.

The instantaneous density contours representing the shock-cell structures are compared in Fig. 11. The diamond 


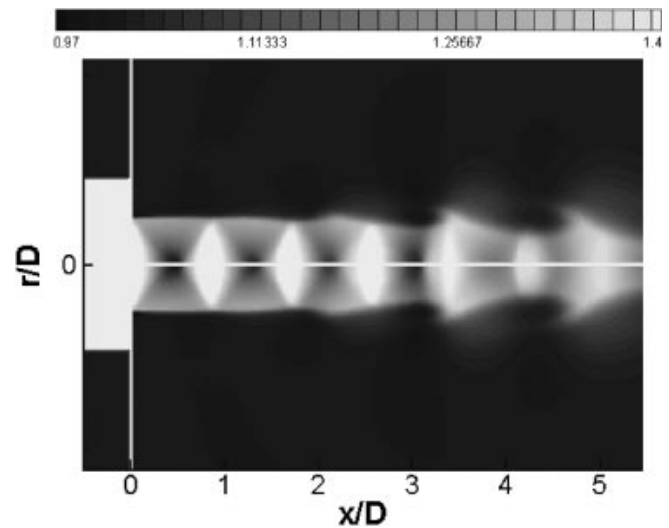

(a) Shape-A

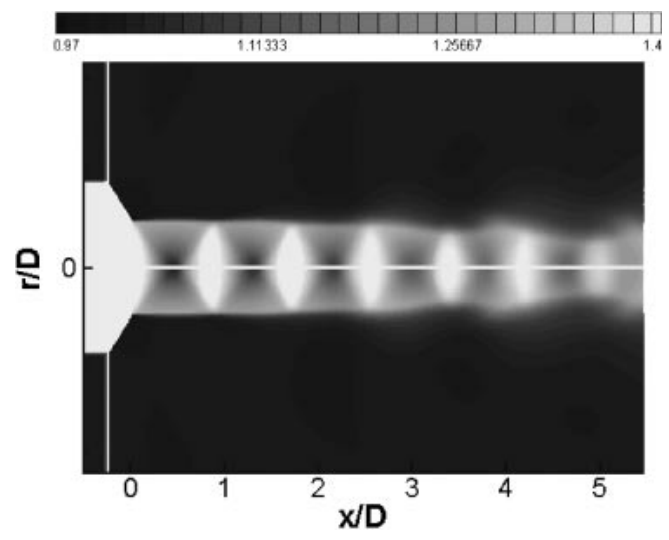

(b) Shape-B

Fig. 11. Comparison of instantaneous density contours showing shockcell structures.

shock-cell structure shape is very important with regard to both screech frequency and amplitude. The computed shock-cell structures have been well produced and are in good agreement with the experimental data by Norum and Brown. ${ }^{13)}$ The first three shock-cells are almost steady, but the shock cells following them are unsteady due to the convection of large-scale vortices. Vorticity contours, which are related to the behavior, are also compared in Fig. 12. For Shape-A, more organized vortices are convected downstream, leading to instability growth.

Pressure signals on the nozzle lip surface are shown in Fig. 13, where $P_{\text {s }}$ represents the static pressure and $P_{\text {a }}$ the ambient pressure. The nondimensional time is defined as $t^{*}=t a_{\infty} / D$, based on the free-stream sound speed and nozzle diameter. The pressure signal obtained on the lip surface indicates change in acoustic pressure, because there is no jet flow there. The screech magnitude on the lip surface of Shape-B is lower than that of Shape-A.

Figure 14 compares pressure distributions along the centerline and in the jet shear layer at $r / D=0.48$. The timeaveraged centerline pressure distribution in Fig. 14(a) is similar, while that of the jet shear layer in Fig. 14(b) is quite different, where the pressure magnitude of shape-B is lower than that of shape-A. Note that the screech tone magnitude is strongly related to the pressure magnitude in the jet shear layer, i.e., the amplitude of instability waves.

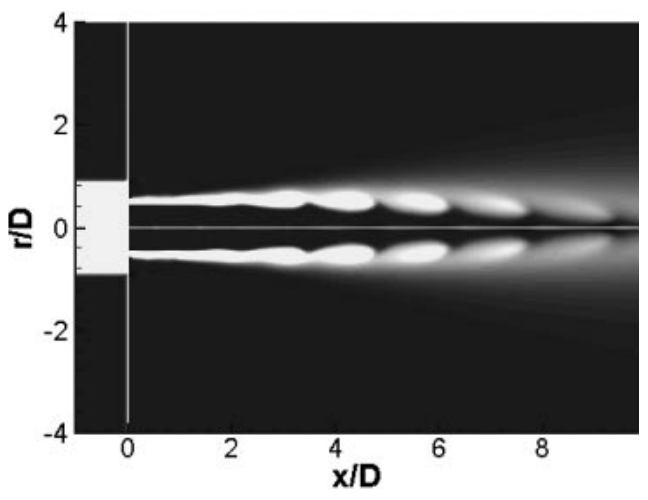

(a) Shape-A: Base geometry

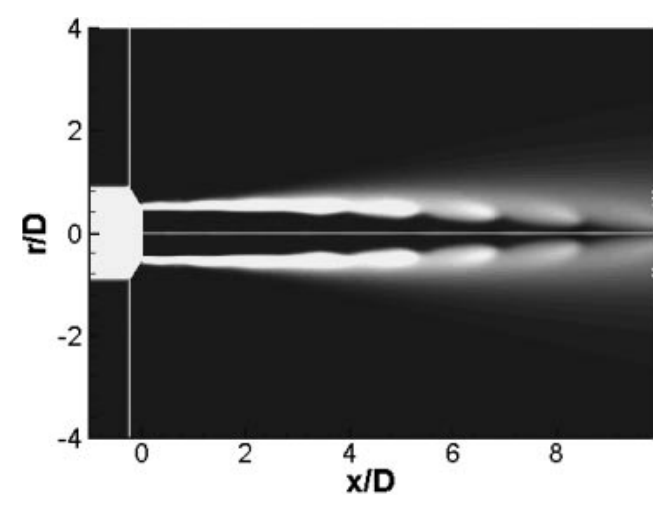

(b) Shape-B: Upward-cut geometry

Fig. 12. Comparison of instantaneous vorticity contours.

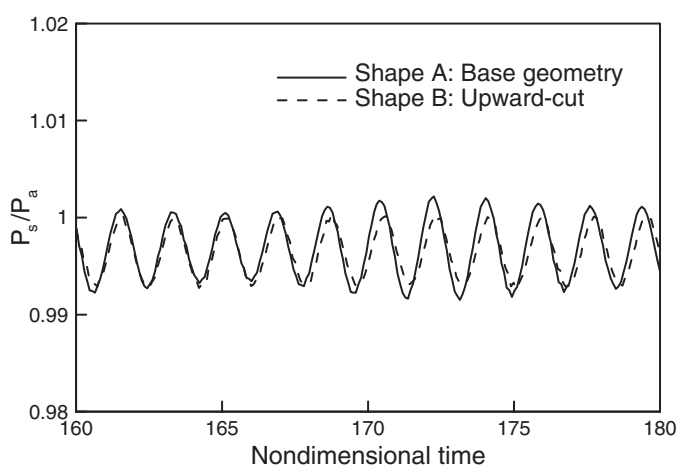

Fig. 13. Comparison of pressure time signal on nozzle lip surface.

Figure 15 compares the sound pressure level between the computed and experimental data. The experiment was conducted by Ponton and Seiner ${ }^{14)}$ who mounted a pressure transducer at a radial distance of $0.642 D$ on the surface of the nozzle lip. Although the experimental SPL trend is somewhat different from the simulated one, the peak levels are nearly equal. Moreover, the present results are in good agreement with Tam's numerical results as jet Mach number increases. The SPL for shape-B is approximately $2 \mathrm{~dB}$ lower than that for shape-A, where the amplitude of reflected and scattered waves along the jet axis is reduced. Thus, the numerical method used in this study can be considered to examine the effect of nozzle lip shape on the amplitude of screech tone. 


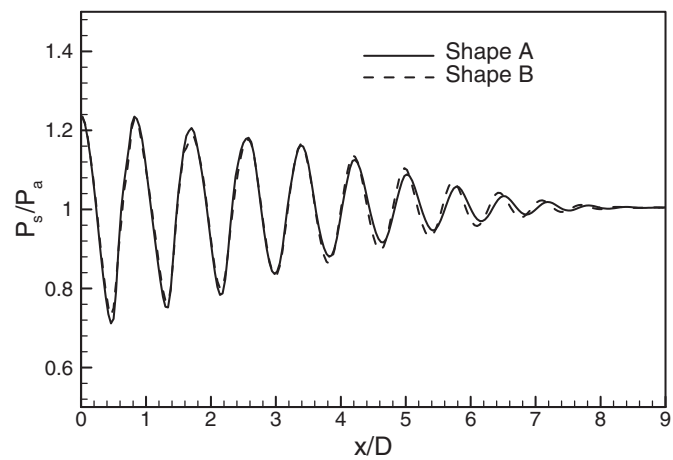

(a) Jet centerline

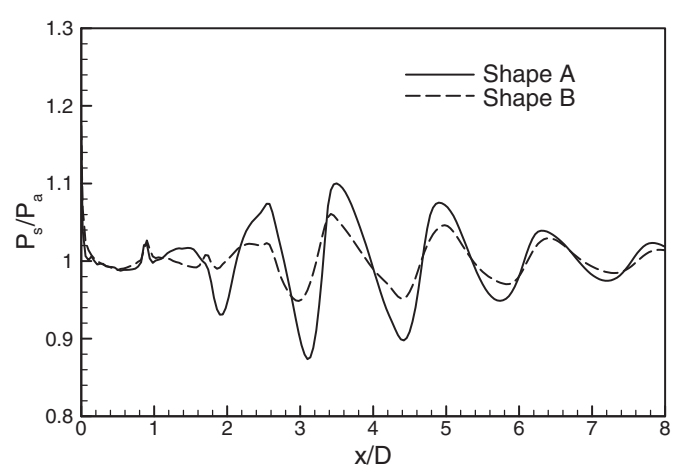

(b) Jet shear layer at $r / D=0.48$

Fig. 14. Pressure distribution along jet axis.

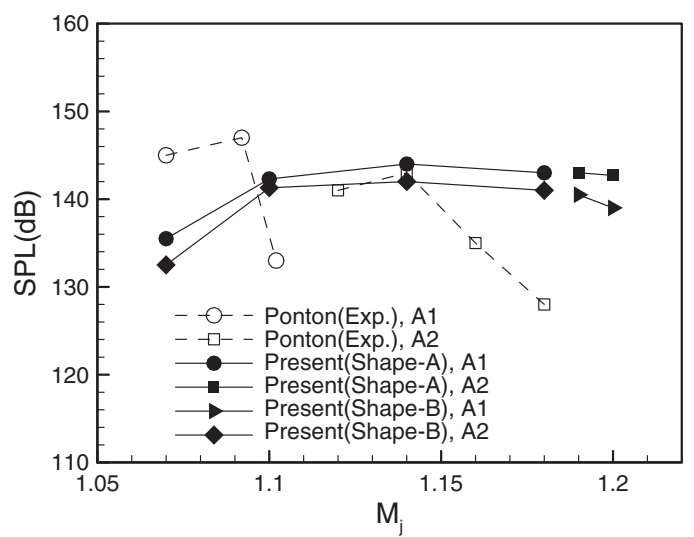

Fig. 15. Comparison of sound pressure level on nozzle lip surface.

\section{Conclusion}

The finite volume Modified-Flux ENO scheme coupled with the characteristic boundary conditions is used to simulate axisymmetric supersonic jet screech tones at different Mach numbers ranging from 1.07 to 1.2. The simulated results for wavelength, mean-flow velocity profile, and pressure distribution along the jet axis agree well with experiment data. The mechanism of screech tone generation was identified by Mach number time variation in the jet plume. Periodic shock motions interacting with instability waves cause compression and expansion of the jet flow, producing the screech tone.

Screeching jet flow was simulated for two nozzle lips; a baseline shape, and an upward-cut shape, to examine the effect of nozzle lip shape on screech tone. Comparison of the screech tone amplitude at the nozzle lip shows that the screech tone for the upper-cut shape is weak compared to that of the baseline shape, because the former suppresses amplitude of reflected waves along the jet axis.

Screech tone magnitude is strongly related to the pressure magnitude in the jet shear layer, i.e., the amplitude of instability waves. In addition, the pressure amplitude in the thin shear layer just after the nozzle exit plays an important role in effectively controlling the magnitude of screech tones.

\section{References}

1) Powell, A.: On the Noise Emanating from a Two-Dimensional Jet above the Critical Pressure, Aeronautical Quarterly, 4 (1953), pp. 103-122.

2) Tam, C. K. W., Shen, H. and Raman, G.: Screech Tones of Supersonic Jets from Bevelled Rectangular Nozzles, AIAA J., 35 (1997), pp. 1119_ 1125 .

3) Shen, H. and Tam, C. K. W.: Numerical Simulation of the Generation of Axisymmetric Mode Jet Screech Tones, AIAA J., 36 (1998), pp. 1801-1807.

4) Loh, C. Y., Hultgren, L. S. and Jorgenson, P. C. E.: Near Field Screech Noise Computation for an Underexpanded Supersonic Jet by the CE/SE Method, AIAA Paper 2001-2252, 2001.

5) Li, X. D. and Gao, J. H.: Numerical Simulation of Axisymmetric Supersonic Screech Tones, AIAA Paper 2004-2951, 2004.

6) Spalart, P. R. and Allmaras, S. R.: A One-Equation Turbulence Model for Aerodynamic Flows, AIAA Paper 1992-0439, 1992.

7) Klaus, A. H.: Computational Fluid Dynamics Volume III, 4th ed., Engineering Education System, Wichita, 2000, pp. 48-50.

8) Kim, Y. S. and Lee, D. J.: Numerical Analysis of Internal Combustion Engine Intake Noise with a Moving Piston and a Valve, J. Sound Vib., 241 (2001), pp. 895-912.

9) Kim, Y. S. and Lee, D. J.: Computation of Shock-Sound Interaction Using Finite Volume Essentially Non-Oscillatory Scheme, AIAA J., 40 (2002), pp. 1239-1240.

10) Thompson, K. W.: Time Dependent Boundary Conditions for Hyperbolic Systems, J. Comput. Phys., 68 (1987), pp. 1-24.

11) Kim, Y. S. and Nakamura, Y.: Characteristic Boundary Conditions with Finite-Volume ENO Scheme for Aeroacoustic Simulations, International Journal of CFD, 21 (2007), pp. 1-10.

12) Lau, J. C.: Effects of Exit Mach Number and Temperatures on Meanflow and Turbulence Characteristics in Round Jets, J. Fluid Mech., 105 (1981), pp. 193-218.

13) Norum, T. D. and Brown, M. C.: Simulated High-Speed Flight Effects on Supersonic Jet Noise, AIAA Paper 1993-4388, 1993.

14) Ponton, M. K. and Seiner, J. M.: The Effects of Nozzle Exit Lip Thickness on Plume Resonance, J. Sound Vib., 154 (1992), pp. 531549.

15) Norum, T. D.: Screech Suppression in Supersonic Jet, AIAA J., 21 (1983), pp. 235-240. 